\title{
Correlation of Atomic Cluster Symmetry and Glass-Forming Ability of Metallic Glass
}

\author{
Xue kui Xi, ${ }^{1}$ Li long $\mathrm{Li},{ }^{1}$ Bo Zhang, ${ }^{2}$ Wei hua Wang, ${ }^{2}$ and Yue $\mathrm{Wu}^{1, *}$ \\ ${ }^{1}$ Department of Physics and Astronomy and Curriculum in Applied and Materials Sciences, University of North Carolina, \\ Chapel Hill, North Carolina 27599-3255, USA \\ ${ }^{2}$ Institute of Physics, Chinese Academy of Sciences, Beijing 100080, China
}

(Received 20 April 2007; published 29 August 2007)

\begin{abstract}
Local structures play a crucial role in glass formation and properties. In addition to topological shortrange order, the geometric property of site symmetry is another important but less known characteristic of local structures. It is shown that the observed sharp increase of glass forming ability of $\mathrm{Ce}_{70-x} \mathrm{Al}_{10} \mathrm{Cu}_{20} \mathrm{Co}_{x}$ upon $\mathrm{Co}$ addition is correlated with a dramatic increase of Al site symmetry, as reflected by decreasing quadrupole frequency measured by ${ }^{27} \mathrm{Al}$ NMR. The result is consistent with the structure model of Al-centered icosahedral clusters as the predominant structural building blocks.
\end{abstract}

DOI: 10.1103/PhysRevLett.99.095501

PACS numbers: 61.18.Fs, 61.43.Fs, 76.60.-k

Fundamental issues and technological importance have inspired continued studies of structures and properties of amorphous solids such as oxide glasses [1], chalcogenides [2,3], gels [4], and metallic glasses [5-7]. The relationship between local structures and the characteristic properties of amorphous materials remains a central theme of current investigations [2,3]. Local structures of metallic glasses are the least understood compared to other amorphous solids $[8,9]$. Scientific interests and technological importance of bulk metallic glasses (BMGs) have stimulated renewed interest in the structure of metallic glasses [5-7]. Recently, solute-centered atomic clusters with favorable short range order have been proposed as the basic building blocks for metallic alloys [5-7]. An example of such clusters is icosahedral cluster with a solute atom at the center and 12 solvent atoms as nearest neighbors. This atomic configuration is a favorable candidate of solutecentered clusters when the ratio of solute versus solvent radius is in the range of 0.8 to 0.9 [10-12]. Correlations between local structures and various properties of metallic glasses have been proposed for both fundamental understanding and applications of metallic glasses [13-15]. For instance, local site symmetry coefficients were proposed to be closely related to local average shear stress, which is relevant for understanding glass-forming ability (GFA) [15]. However, site symmetry in glassy state is inaccessible for conventional diffraction techniques. Nuclear magnetic resonance (NMR) is potentially useful for probing such characteristics of local structures [16-19].

$\mathrm{CeCuAl}(\mathrm{Co})$ bulk glass formers [20] with strong composition effects on GFA have been selected for investigating characteristics of local structures in BMGs. Here, Al atoms are considered to be the solute atoms. Since ${ }^{27} \mathrm{Al}$ is a quadrupolar nucleus, the electric-field gradient (EFG) at the $\mathrm{Al}$ site can be determined by ${ }^{27} \mathrm{Al} \mathrm{NMR}$. EFG is related to the site symmetry coefficients since both are secondrank tensors and both vanish under cubic and higher local symmetries such as icosahedral symmetry. In this Letter, a systematic study of the $\mathrm{CeCuAl}(\mathrm{Co})$ bulk glass formers using ${ }^{27} \mathrm{Al} \mathrm{NMR}$ was performed. An evolution of cluster symmetry, as reflected by changes of EFG upon a small change of Co concentration, was observed in the glassy state. In addition, characteristics of bonding between $\mathrm{Al}$ and neighboring atoms were also identified. Compared to previous molecular dynamic simulation and neutron scattering observations [6,7], this work provides an important insight into the nature of the local structure around solute atoms and its role on GFA of metallic glasses.

Three glassy $\mathrm{Ce}_{70-x} \mathrm{Cu}_{20} \mathrm{Al}_{10} \mathrm{Co}_{x}$ samples with $x=0$, 0.2 , and 1 were fabricated by a conventional copper mould casting process. The amorphous nature was characterized by $\mathrm{x}$-ray diffraction, TEM, and confirmed by differential scanning calorimetry. All NMR measurements were performed in a magnetic field of $7.01 \mathrm{~T}$. Nutation experiments were employed to determine the EFG at the Al sites [2124]. Here, the pulse sequence used is $\left(t_{1}\right)_{x}-\tau_{1}-\left(t_{2}\right)_{x}-$ $\tau_{2}-$ acquisition $_{-y}$, where $t_{1}$ and $t_{2}$ are pulse widths, $x$ and $-y$ are pulse and receiver phases, respectively, and $\tau_{1}$ and $\tau_{2}$ are time delays. The echo signal appearing at $\tau_{2}=\tau_{1}$ was recorded and the signal intensity associated with the central transition $|-1 / 2\rangle \leftrightarrow|1 / 2\rangle$ was then determined as a function of $t_{1}$ with fixed $t_{2}$ and $\tau_{1}$ values. The rf pulse strength $\omega_{\text {rf }}$ was calibrated using several methods. $1 \mathrm{M}$ $\mathrm{Al}\left(\mathrm{NO}_{3}\right)_{3}$ aqueous solution was used to determine the $\pi / 2$ pulse width $\omega_{\mathrm{rf}} t_{\pi / 2}=\pi / 2$. Careful measurements of quality factors were used to estimate the $\omega_{\text {rf }}$ with metallic glass samples. The $\omega_{\text {rf }}$ value was also measured with metallic glass samples by using weaker and selective pulses; the $\omega_{\text {rf }}$ value corresponding to strong rf was calculated by scaling with the ratio of the strong and weak pulse readings recorded through a directional coupling by an oscilloscope. Although various rf strengths were explored in nutation experiments, $\omega_{\mathrm{rf}} / 2 \pi=51 \mathrm{kHz}$ was selected for the systematic nutation measurements based on the range of EFG values involved. The acquisition parameters are $\tau_{1}=$ $14 \mu \mathrm{s}, t_{2}=5 \mu \mathrm{s}$, and $t_{1}$ varies from 0 to $6 \mu \mathrm{s}$ in an increment of $0.5 \mu \mathrm{s}$. Signal averaging was carried out with a recycle delay of $150 \mathrm{~ms}$. Magnetic susceptibilities 


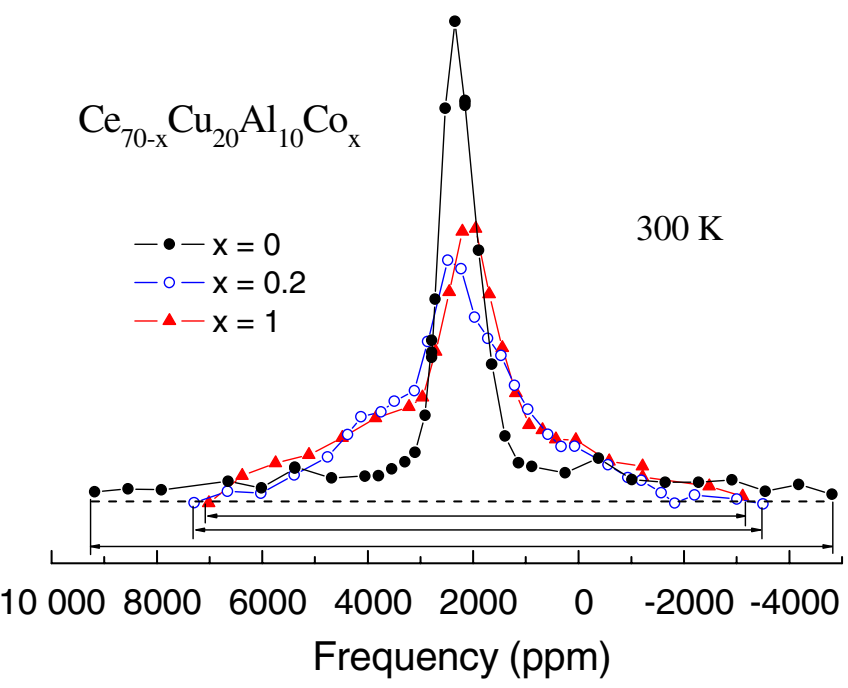

FIG. 1 (color online). ${ }^{27} \mathrm{Al}$ point-by-point spectra of $\mathrm{Ce}_{70-x} \mathrm{Al}_{10} \mathrm{Cu}_{20} \mathrm{Co}_{x}(x=0,0.2,1)$ obtained by the Hahn-echo point-by-point method. The shift reference is $1 \mathrm{M} \mathrm{Al}\left(\mathrm{NO}_{3}\right)_{3}$ aqueous solution. The solid lines are a guide for the eye. The widths of the satellite spectra, proportional to $V_{z z}$ or $\omega_{Q} / 2 \pi$, are marked with arrows to show the big change of quadrupole frequency with a small amount of Co addition.

of samples were measured with a quantum design SQUID magnetometer in the temperature range of 80 to $300 \mathrm{~K}$ in a magnetic field of $5 \mathrm{~T}$.

Figure 1 shows ${ }^{27} \mathrm{Al}$ NMR spectra of the three samples with $x=0,0.2$, and 1 at room temperature (RT). ${ }^{27} \mathrm{Al}$ is a spin $I=5 / 2$ nucleus and its spectrum consists of five $|m\rangle \leftrightarrow|m+1\rangle$ transitions including the narrow $|-1 / 2\rangle \leftrightarrow|1 / 2\rangle$ central transition, broadened only by the second-order quadrupole effect, and wide satellite transitions $|m\rangle \leftrightarrow|m+1\rangle(m \neq-1 / 2)$. The satellite transitions are broadened by the first-order quadrupole interaction given by [21]

$H_{Q}=\frac{1}{12} \omega_{Q}\left[3 I_{z}^{2}-I(I+1)\right]\left(3 \cos ^{2} \beta-1+\eta \sin ^{2} \beta \cos 2 \alpha\right)$,

where $\omega_{Q}=\frac{3 e^{2} q Q}{2 I(2 I-1) \hbar}$ is the quadrupole frequency, $Q$ is the electric quadrupole moment, eq $=V_{Z Z}$ is the $Z$ principal component of the EFG tensor which vanishes under cubic or higher symmetries, $\eta$ is the asymmetry parameter of the EFG tensor, $\alpha$ and $\beta$ are Euler angles which relate the principal axis system of the EFG tensor to the laboratory frame, and $I_{z}$ is the nuclear spin operator along the external magnetic field direction z. Since $\alpha$ and $\beta$ are distributed randomly in an amorphous system, anisotropic broadening occurs giving rise to the broad satellite transitions. Figure 1 shows that the most notable change of the spectra upon Co addition is the drastic narrowing of the satellite transitions even at very low Co content of 0.2 at. \%. The narrowing leads to the amplitude increase of the satellite transitions.

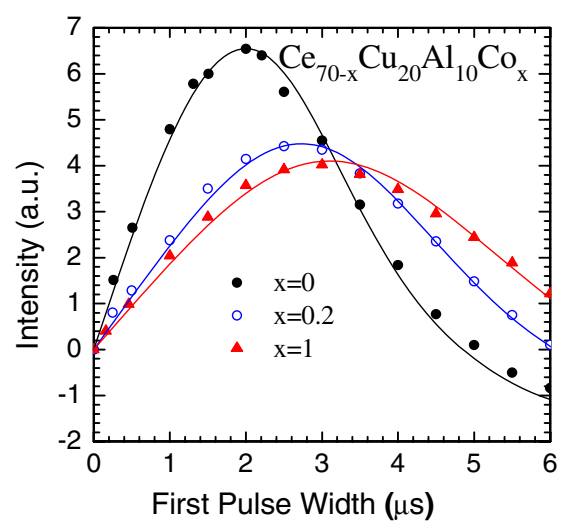

FIG. 2 (color online). Nutation experiments where the central transition intensity is plotted versus the first pulse duration in a Hahn-echo sequence. The pulse duration $t_{1}$ increases from 0 to $6 \mu \mathrm{s}$ by steps of $0.5 \mu \mathrm{s}$. The amplitude of the $\mathrm{rf}$ pulse is $\omega_{\text {rf }} / 2 \pi=51 \mathrm{kHz}$ for all three samples. Solid lines are fittings with $\omega_{Q}$ and $\eta$ as parameters as described in the text. The fitting results are summarized in Table I.

Equation (1) shows that this corresponds to a significant reduction of $\omega_{Q}$, thus $V_{Z Z}$ of the EFG tensor. Nutation experiments were conducted to confirm and quantify the decrease of EFG upon Co addition. Figure 2 shows the central transition intensity versus the width of the first pulse $t_{1}$ ranging from 0 to $6 \mu \mathrm{s}$ for all three samples. The evolution of the spin density operator $\rho\left(t_{1}, \tau_{1}, t_{2}, \tau_{2}\right)$, thus the signal, under the Hamiltonian $H=\omega_{\mathrm{rf}} I_{x}+H_{Q}$ was calculated numerically including powder averaging with given values of $\omega_{Q}$ and $\eta$ for each $t_{1}$ value [24]. The calculated nutation curve is then fit with the experimental data with $\omega_{Q}$ and $\eta$ as fitting parameters. The best fits are shown in Fig. 2 and the corresponding fitting values of $\omega_{Q}$ and $\eta$ are listed in Table I. As expected, $\omega_{Q} / 2 \pi$ decreases dramatically from $600 \mathrm{kHz}$ in the $x=0$ sample to $120 \mathrm{kHz}$ in the $x=1$ sample. Both nutation results and the change of satellite linewidth prove clearly that there is significant change of local structure upon microalloying and the symmetry around $\mathrm{Al}$ sites is enhanced significantly upon Co addition.

To better understand the local structural feature around $\mathrm{Al}$ sites and the effect of microalloying, the electronic and

TABLE I. Fitting values of quadrupole frequency, $\omega_{Q} / 2 \pi$, and asymmetry parameter, $\eta$, of the nutation experiments along with melting temperature $T_{m}$ and critical diameter $D_{c}$.

\begin{tabular}{lccccc}
\hline \hline \multicolumn{1}{c}{ Composition } & $\begin{array}{c}\omega_{Q} / 2 \pi \\
(\mathrm{kHz})\end{array}$ & $\eta$ & $\begin{array}{c}\text { Density } \\
\left(\mathrm{g} / \mathrm{cm}^{3}\right)\end{array}$ & $\begin{array}{c}T_{m} \\
(\mathrm{~K})\end{array}$ & $\begin{array}{c}\text { Critical } \\
\text { diameter } \\
\left(D_{c}, \mathrm{~mm}\right)\end{array}$ \\
\hline $\mathrm{Ce}_{70} \mathrm{Cu}_{20} \mathrm{Al}_{10}$ & 600 & 0.9 & 6.669 & 647 & 2 \\
$\mathrm{Ce}_{69.8} \mathrm{Cu}_{20} \mathrm{Al}_{10} \mathrm{Co}_{0.2}$ & 180 & 0.8 & 6.733 & 643 & 8 \\
$\mathrm{Ce}_{69} \mathrm{Cu}_{20} \mathrm{Al}_{10} \mathrm{Co}_{1}$ & 120 & 0.8 & 6.753 & 615 & 10 \\
\hline \hline
\end{tabular}


magnetic properties were also investigated by NMR. It is interesting to note that the observed resonance shift of about $2300 \mathrm{ppm}$ at RT, as shown in Fig. 1, is much larger than the Knight shift of 1630 ppm observed in pure Al. However, further analysis shows that this shift can be attributed almost entirely to the Knight shift associated with transferred hyperfine interactions with local magnetic moments of trivalent $\mathrm{Ce}$ atoms $\left(\mathrm{Ce}^{3+}\right)$ [25]. Figure 3(a) shows the measured molar magnetic susceptibility which clearly follows the Curie-Weiss law $\chi_{\mathrm{mol}}^{\mathrm{CW}}=C /(T-\theta)$ down to $130 \mathrm{~K}$. Fittings yield Weiss temperature $\theta=$ $-(43 \pm 5) \mathrm{K}$ for $x=0$ and $\theta=-(58 \pm 5) \mathrm{K}$ for $x=1$. The measured Curie constant $C=0.72 \mathrm{emu} \cdot \mathrm{K} / \mathrm{Oe}$. mol-Ce for $x=0$ and $C=0.87 \mathrm{emu} \cdot \mathrm{K} / \mathrm{Oe} \cdot \mathrm{mol}-\mathrm{Ce}$ for $x=1$ yield an effective magnetic moment of $2.40 \mu_{B}$ and $2.64 \mu_{B}$, respectively, for $x=0$ and 1 . This is very close to the trivalent $\mathrm{Ce}^{3+}$ free-ion value of $2.54 \mu_{B}$. Figure 3(b) shows the linear dependence of ${ }^{27} \mathrm{Al}$ NMR isotropic shift versus magnetic susceptibility. The shift extrapolated to $\chi_{\mathrm{mol}}^{\mathrm{CW}}=0$ is $190 \pm 40 \mathrm{ppm}$ for $x=0$ and $50 \pm 40$ ppm for $x=1$.

The isotropic shift can be described by $K=K_{o}+K_{s}+$ $K_{s f}$, where $K_{o}$ represents the orbital contribution comparable to typical ${ }^{27} \mathrm{Al}$ chemical shifts, $K_{s}$ is the Knight shift due to the contact hyperfine interaction with $s$ conduction electrons at the Fermi level given by $K_{s}=(8 \pi / 3) \times$ $\left\langle|\psi(0)|^{2}\right\rangle_{E_{F}} \Omega \chi_{\text {Pauli }}$, where $\left\langle|\psi(0)|^{2}\right\rangle_{E_{F}}$ is the density of the electron wave function at the nucleus averaged over all states at the Fermi level $E_{F}$ and is normalized over an arbitrarily chosen volume $\Omega$. $\chi_{\text {Pauli }}$ is the Pauli paramagnetic volume susceptibility [26]. $K_{s f}$ is the Knight shift associated with the transferred hyperfine interactions with local magnetic moments of neighboring $\mathrm{Ce}$ atoms due to $s-f$ exchange. The term $K_{s f}$ is given by $K_{s f}=$ $\left(z H_{\mathrm{hf}}^{s f} / \mu_{B} N_{A}\right) \chi_{\mathrm{mol}}^{\mathrm{CW}}$, where $H_{\mathrm{hf}}^{s f}$ is the transferred hyperfine field, $z$ is the number of Ce nearest neighbors to $\mathrm{Al}$, and $N_{A}$ is the Avogadro's number. Fittings yield $z H_{\mathrm{hf}}^{s f}=5.6 \mathrm{kOe}$ (a)

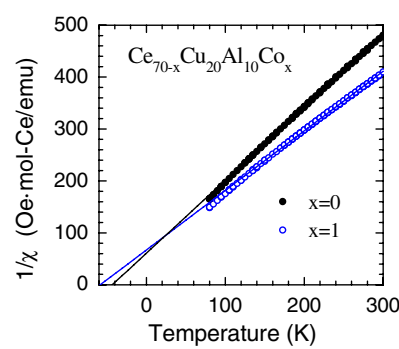

(b)

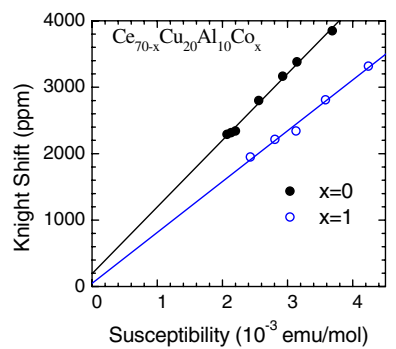

FIG. 3 (color online). (a) Temperature dependences of measured molar magnetic susceptibility (corrected for diamagnetism). Solid lines are the least-square linear fits down to $130 \mathrm{~K}$. (b) Knight shifts versus magnetic susceptibility with temperature as an implicit parameter. Solid lines are the least-square fits using the Curie-Weiss law. for $x=0$ and $z H_{\mathrm{hf}}^{s f}=4.3 \mathrm{kOe}$ for $x=1 . K_{o}+K_{s}$ is associated with the observed shift extrapolated to $\chi_{\mathrm{mol}}^{\mathrm{CW}}=$ 0 . The very small values of $190 \mathrm{ppm}$ for $x=0$ and $50 \mathrm{ppm}$ for $x=1$, as compared to $1630 \mathrm{ppm}$ in pure $\mathrm{Al}$ metal, are strong indications that there is almost no $s$ character at $\mathrm{Al}$ sites in the electron wave functions at the Fermi level. One of the possible reasons is the presence of a pseudogap at the Fermi level in electron wave functions projected at $\mathrm{Al}$ sites. It is very interesting to note that nearly all $\mathrm{Al}$ icosahedral alloys show a very small Knight shift for ${ }^{27} \mathrm{Al}$ NMR [27]. The very small Knight shifts associated with the Pauli susceptibility suggest that bonding of $\mathrm{Al}$ atoms with neighboring atoms could have strong covalent characters as compared to free electrons and possibly opening a pseudogap for electron wave functions projected at $\mathrm{Al}$ sites. In addition to the subtle changes of $z H_{\mathrm{hf}}^{s f}$ and $K_{o}+K_{s}$, the RT spin-lattice relaxation time $T_{1}$ also changes from $20 \mathrm{~ms}$ for $x=0$ to $50 \mathrm{~ms}$ for both $x=0.2$ and 1 . These observed changes show that Co addition causes subtle changes of electronic states.

Insight can be gained on the evolution of microalloyinginduced cluster symmetry and its implication on GFA by considering the role of atomic size ratio and bonding nature between solute and solvent atoms. The optimal atomic size ratio between solute and solvent atoms is crucial for efficient atomic packing and potential energy minimization in both liquid and glassy state $[7,10,12]$. Using metallic atomic radii $[10,11] r_{(\mathrm{Al})}=1.43 \AA, r_{(\mathrm{Cu})}=1.28 \AA$, and $r_{(\mathrm{Ce})}=1.82 \AA$, and considering the composition of $\mathrm{Ce}_{70} \mathrm{Al}_{10} \mathrm{Cu}_{20}$, the estimated ratio $r_{(\mathrm{Al})} / r_{(\mathrm{Ce}, \mathrm{Cu})}$ is around 0.84 . From an efficient packing point of view, this ratio is close to 0.902 based on hard sphere model $[10,11]$ and 0.85 based on minimum energy consideration [12], indicating that icosahedralike Al-centered clusters could be the predominant cluster configurations in the sample. This argument is also in agreement with the fact that ${ }^{27} \mathrm{Al} \mathrm{Knight}$ shift is very small, i.e. $\sim 200 \mathrm{ppm}$ in nearly all icosahedral Al-bearing alloys [27], while the isotropic Knight shifts in typical Al bearing non-glass-forming alloys is much larger, such as $1480 \mathrm{ppm}$ in $\mathrm{Al}_{2} \mathrm{Cu}$ [28]. When small amount of $\mathrm{Co}$ is added, the bulk density increases, as shown in Fig. 4, along with further decrease of the Knight shift and the spin-lattice relaxation rates $\left(1 / T_{1}\right)$. These results suggest that the atomic ratio could have approached closer to the optimal value for local icosahedral symmetry at Al sites due to the subtle change of electronic structure and leads to less local strains. Theoretical calculation shows that when the effective size ratio is close to 0.85 , the potential energy of icosahedral cluster approaches minimum in metallic glasses [12], consistent with our experimental observations. Such improved site symmetry upon Co addition should have important implications for the stability of both liquid and glassy states $[10,11]$. As listed in Table I, the melting temperature $\left(T_{m}\right)$ drops from 647 to $643 \mathrm{~K}$ by adding 0.2 at. $\%$ Co to $\mathrm{Ce}_{70-x} \mathrm{Al}_{10} \mathrm{Cu}_{20} \mathrm{Co}_{x}$ and to $615 \mathrm{~K}$ by 


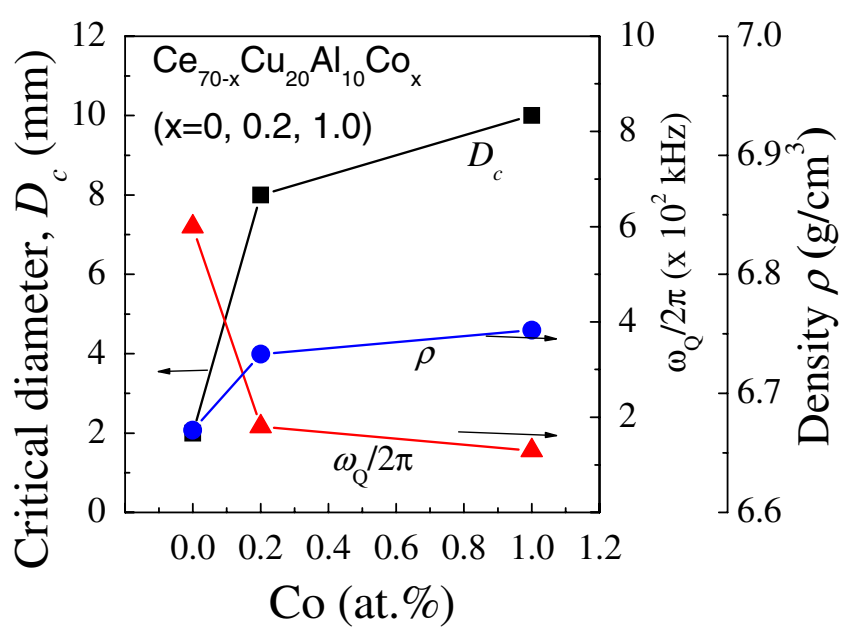

FIG. 4 (color online). (a) Critical diameter $\left(D_{c}\right)$, quadrupole frequency $\left(\omega_{Q} / 2 \pi\right)$, and bulk density $(\rho)$ as function of Co concentration, showing clear correlation between GFA and $\omega_{Q} / 2 \pi$.

adding 1 at. \% Co. The decrease of melting temperature shows explicitly that the more symmetric clusters (or topological ordering configurations) correspond to the deeper eutectic point. Figure 4 shows that the increase of critical diameter $D_{c}$, which is widely used as one of the convenient and reliable parameter to evaluate GFA [29], correlates very well with the decrease of quadrupole frequency $\omega_{Q} / 2 \pi$. This correlation is clear evidence that the origin of microalloying effect on GFA is the change of local structures [30].

In conclusion, we used ${ }^{27} \mathrm{Al} \mathrm{NMR}$ to explore systematically the local structure of $\mathrm{CeCuAl}(\mathrm{Co})$ bulk metallic glass at various compositions. An evolution of cluster symmetry upon systematic small tuning of Co concentration has been observed as reflected by changes of EFG. Characteristics of bonding between $\mathrm{Al}$ and neighboring atoms are also identified and are consistent with icosahedral clusters. The results provide important insight into the nature of local structures around solute atoms and its intrinsic role on GFA in metallic glasses. This work also sheds light on the local structure of deep, higher-order "eutectic" liquid [9].

This work was supported by the U.S. Army Research Office (Grant no. W911NF-05-1-0342).

*Corresponding author. yuewu@physics.unc.edu
[1] Q. Mei, C. J. Benmore, and J.K. R. Weber, Phys. Rev. Lett. 98, 057802 (2007).

[2] A. L. Greer and N. Mathur, Nature (London) 437, 1246 (2005).

[3] A. V. Kolobov et al., Phys. Rev. Lett. 97, 035701 (2006).

[4] A. D. Dinsmore, V. Prasad, I. Y. Wong, and D. A. Weitz, Phys. Rev. Lett. 96, 185502 (2006).

[5] D. B. Miracle, Nat. Mater. 3, 697 (2004).

[6] W. K. Luo, S. W. Sheng, F. M. Alamgir, J. M. Bai, J. H. He, and E. Ma, Phys. Rev. Lett. 92, 145502 (2004).

[7] S. W. Sheng, W. K. Luo, F. M. Alamgir, J. M. Bai, and E. Ma, Nature (London) 439, 419 (2006).

[8] S. R. Elliott, Physics of Amorphous Materials (Longman Group Ltd., London, 1990), 2nd ed.

[9] W. L. Johnson, MRS Bull. 24, 42 (1999).

[10] T. Egami and Y. Waseda, J. Non-Cryst. Solids 64, 113 (1984).

[11] D. B. Micracle, W. S. Sanders, and O. N. Senkov, Philos. Mag. 83, 2409 (2003).

[12] H. J. Lee, T. Cagin, W. L. Johnson, and W. A. Goddard, III, J. Chem. Phys. 119, 9858 (2003).

[13] S. G. Mayr, Phys. Rev. Lett. 97, 195501 (2006).

[14] Y. Zhang and A. L. Greer, Appl. Phys. Lett. 89, 071907 (2006).

[15] D. Srolovitz, K. Maeda, V. Vitek, and T. Egami, Philos. Mag. A 44, 847 (1981).

[16] P. Panissod et al., Phys. Rev. Lett. 44, 1465 (1980).

[17] X. P. Tang, J. F. Loffler, W. L. Johnson, and Y. Wu, J. NonCryst. Solids 317, 118 (2003).

[18] H. Breitzke, K. Luders, S. Scudino, U. Kuhn, and J. Eckert, Phys. Rev. B 70, 014201 (2004).

[19] M. Imafuku et al., J. Non-Cryst. Solids 351, 3587 (2005).

[20] B. Zhang, R. J. Wang, D. Q. Zhao, M. X. Pan, and W. H. Wang, Phys. Rev. B 73, 092201 (2006).

[21] P. P. Man, E. Duprey, J. Fraissard, P. Tougne, and J.-B. d'Espinose, Solid State Nucl. Magn. Reson. 5, 181 (1995).

[22] A. Samoson and E. Lippmaa, Phys. Rev. B 28, 6567 (1983).

[23] A. P. M. Kentgens, J. J. M. Lemmens, F. M. M. Geurts, and W. S. Veeman, J. Magn. Reson. 71, 62 (1987).

[24] P. P. Man, Phys. Rev. B 52, 9418 (1995).

[25] J. M. Lawrence, P. S. Riseborough, and R. D. Parks, Rep. Prog. Phys. 44, 1 (1981).

[26] A. Narath, in Hyperfine Interactions, edited by A.J. Freeman and R.B. Frankel, Nuclear Magnetic Resonance in Magnetic and Metallic Solids (Academic, New York and London, 1984).

[27] E. A. Hill et al., Phys. Rev. B 49, 8615 (1994).

[28] T. J. Bastow and S. Celotto, Acta Mater. 51, 4621 (2003).

[29] X.H. Lin and W.L. Johnson, J. Appl. Phys. 78, 6514 (1995).

[30] W. H. Wang, Prog. Mater. Sci. 52, 540 (2007). 